\title{
OPEN TOPICS IN FUZZY COALITIONAL GAMES WITH TRANSFERABLE UTILITY
}

\author{
MILAN MAREŠ \\ Institute of Information Theory and Automation \\ Academy of Sciences of the Czech Republic \\ P.O. Box 18, 18208 Praha 8, Czech Republic \\ E-mail:mares@utia.cas.cz
}

I had the pleasure to meet Jerzy Łos and cooperate with him in 1972, and to be in contact with him in the following years. The cooperation meant for me a meeting with a strong scientific as well as human personality with exactly formulated and deeply thought-out approach to the sense of mathematics and life. My professional orientation was influenced by that meeting, and it is a great honour for me to contribute to this volume.

Author

\begin{abstract}
Vagueness is one of the phenomena which cannot be separated from the real bargaining and cooperative situations. The aim of this paper is to offer a brief survey of the recent state-of-art of the modelling of vagueness in coalitional games with transferable utility. It may be recognized in two components of these games, namely, in vague structure of coalitions where each player may simultaneously participate in several of them, and in vague expectations of coalitional pay-offs. Both these cases are described in the paper and approaches to including them into the game model are analyzed. An attempt to unify both cases into one uniform model is discussed as well.
\end{abstract}

1. Introduction. The bargaining and coalitions forming in real cooperative situations is usually connected with some kind of vagueness following from the fact that the agreements about cooperation are accepted before the real run of the game, and that also the expectations of pay-offs and mutual relations among particular players are often connected with subjectivity. These facts are not included in the classical models of coop-

2000 Mathematics Subject Classification: Primary 91A12; Secondary $03 E 72$.

Key words and phrases: game, coalition, TU-game, fuzzy coalition, fuzzy pay-off, fuzzy class of games.

This paper includes and analyzes some results and ideas developed under the support of the Grant Agency of the Czech Republic (grant No. 402/04/1026), Grant Agency of the Academy of Sciences of CR (No. A 1075301) and Project of Research Centre (No. 1M0572).

The paper is in final form and no version of it will be published elsewhere. 
eration and corresponding solution concepts (cf. $[20,19,7])$. Some attempts to treat the phenomenon of uncertainty into the coalitional game theory were done with the development of fuzzy set theory, and they continue till now. This paper aims to offer a brief overview of the recent state-of-art in this endeavour, and to discuss some open problems still existing in this field.

We limit our attention to the model of games with transferable utility (TU-games) which is simpler and more lucid. Nevertheless, it is useful to keep in mind that some of the suggested models (see, e.g. $[1,2,15]$ ) are suitable for the games with non-transferable utility (NTU-games), as well.

After a brief presentation of the very elementary concepts and notations of both, TU-games and fuzzy sets, the main section is devoted to particular models of fuzziness in cooperative games. The considered deterministic model of game is very simple-it consists of two concepts, coalition and coalitional pay-off (characteristic function), and both of them can be fuzzified. The fuzzification of coalitions is based on the fact that many real players may participate in more than one coalition, parallelly. They distribute their activity among several groups of partners and cooperate with them with different intensity. The first models of games in which the coalitions are fuzzy subsets of the set of all players, were suggested in seventieth (see, e.g. [1, 2]), and they are developed till now $([3,11,17])$. The fuzzification of the pay-offs was suggested in the nineties and the first results were summarized in [10]. Also this model was developed and some modifications were suggested (cf. [16]). An elementary attempt to suggest the fuzzy modification of cooperative market equilibrium was done in [12].

In the main part of this paper the particular attempts to the fuzzification of TU-games are discussed and some of the still open problems connected with them are mentioned. Moreover, the possibility of construction of a universal fuzzy TU-game covering both types of vagueness is considered and its eventual attributes are analyzed.

If $M$ is a set then we denote, in the whole paper, by $\mathcal{P}(M)$ the class of all subsets of $M$.

2. Coalitional game with transferable utility. Let us recall, very briefly, that a TU-game is defined by a pair $(I, v)$, where $I=\{1,2, \ldots, n\}$ is the set of players and $v: \mathcal{P}(I) \rightarrow R$, where $R$ is the set of real numbers, is the characteristic function. Every set of players $K \subset I$ is a coalition and the characteristic function value $v(K)$ is the expected total pay-off of coalition $K$. We suppose that for the empty coalition $\emptyset, v(\emptyset)=0$. Every real-valued vector $\boldsymbol{x}=\left(x_{1}, x_{2}, \ldots, x_{n}\right) \in R^{n}$ is called an imputation.

The game is said to be superadditive iff for every pair of disjoint coalitions $K, L$ $v(K \cup L) \geq v(K)+v(L)$, and it is called convex iff for every pair of coalitions $v(K \cup L)+$ $v(K \cap L) \geq v(K)+v(L)$.

The core of the game $(I, v)$ is a set of imputations

$$
\mathcal{C}_{v}=\left\{\boldsymbol{x} \in R^{I}: \sum_{i \in I} x_{i} \leq v(I) \text { and } \forall K \subset I, \sum_{i \in K} x_{i} \geq v(K)\right\} .
$$

All these concepts are elementary in the theory of games (see, e.g., $[19,7,20])$ and they were recalled here to fix the notations. 
3. Fuzzy set and fuzzy quantity. The concepts of fuzzy set theory will be used, here, for modelling the vagueness of some components of TU-games. Let us recall the seminal paper [21] and many others among which [8, 4, 6, 5] will be especially referred to in the following sections.

If $\mathcal{U}$ is a non-empty set (sometimes called universe), then its fuzzy subset $A$ is defined by a membership function $\mu_{A}: \mathcal{U} \rightarrow[0,1]$. The membership function $\mu_{A}$ is interpreted as a generalization of the characteristic function $\varphi_{A}$ of the deterministic (also called crisp) set $A \subset \mathcal{U}$, where for each $u \in \mathcal{U}, \varphi_{A}(u)=0$ iff $u \notin A$ and $\varphi_{A}(u)=1$ iff $u \in A$. In the fuzzy set theory, for every $u \in \mathcal{U}, \mu_{A}(u)=0$ if $u$ certainly does not belong to $A, \mu_{A}(u)=1$ if $u$ belongs to $A$ without any doubts, and $\mu_{A}(u) \in(0,1)$ if the membership of $u$ in $A$ is vague in any sense (subjective estimation, imprecision of data, unspecified verbal characteristics, noise, imperfect knowledge, etc.) Operations with fuzzy sets (complement, union, intersection,... ) extend the analogous operations with crisp sets (see, e.g. $[21,8,9,5]$ and many others). For any universum $\mathcal{U}$ we denote by $\mathcal{F}(\mathcal{U})$ the set of all fuzzy subsets of $\mathcal{U}$.

If we put the set of real numbers $R$ for the universum then its fuzzy subset $a$ with membership function $\mu_{a}: R \rightarrow[0,1]$ is called a fuzzy quantity if

- $\mu_{a}\left(x_{A}\right)=1$ for some $x_{a} \in R$.

- there exist $x_{1}, x_{2} \in R, x_{1}<x_{a}<x_{2}$, such that $\mu_{a}(x)=0$ for all $x \notin\left[x_{1}, x_{2}\right]$.

Fuzzy quantities represent the vagueness of quantitative data, typical for many practical applications. They can be processed by algebraical operations extending the classical operations with deterministic (crisp) numbers by means of so called extension principle (see, e.g., $[4,8,9,5]$ ). For our purpose, we need the operations of addition and product with a crisp number. If $a, b$ are fuzzy quantities with membership functions $\mu_{a}, \mu_{b}$, respectively, and if $r \in R$ is a real number, then the sum $a \oplus b$ and crisp product $r \cdot a$ are fuzzy quantities, too, with membership functions

$$
\begin{gathered}
\mu_{a \oplus b}(x)=\sup \left[\min \left(\mu_{a}(y), \mu_{b}(x-y)\right)\right], \\
\mu_{r \cdot a}(x)=\mu_{a}(x / r) \text { for } r \neq 0, \quad \mu_{0 \cdot a}(0)=1, \quad \mu_{0 \cdot a}(x)=0 \text { if } x \neq 0,
\end{gathered}
$$

for any $x \in R$.

The properties of these operations are summarized e.g. in $[8,9]$. Let us stress that they are not identical with the algebraical properties of deterministic numbers.

The TU-games theory frequently uses the comparison of numerical values. In some of their fuzzified modifications dealing with fuzzy pay-offs we have to compare fuzzy quantities. As their values are vague, the validity of ordering relations between them will be vague, as well. It means that fuzzy inequality $\succeq$ is a fuzzy relation over $\mathcal{F}(R)$ which is formally represented by a fuzzy subset of the Cartesian product $\mathcal{F}(R) \times \mathcal{F}(R)$ with membership function $\nu_{\succeq}: \mathcal{F}(R) \times \mathcal{F}(R) \rightarrow[0,1]$. For every pair of fuzzy quantities $a, b$ the value $\nu_{\succeq}(a, b)$ denotes the possibility $a \succeq b$, and it is defined by

$$
\nu_{\succeq}(a, b)=\sup \left[\min \left(\mu_{a}(x), \mu_{b}(y)\right): x, y \in R, x \geq y\right] .
$$

4. Fuzzification of TU-games. Even if the classical model of TU-game is deterministic, in many real cooperative situations its both components, the coalitions and their 
expectations of pay-offs, are connected with some vagueness. In this section, we briefly summarize the main approaches to their fuzzification. In the whole section, we preserve the notations used in Section 2.

4.1. Fuzzy coalitions. In the real cooperative situations, each player can participate (and usually does) in more than one coalition (investor can allocate his capital in more companies, researcher may participate in several projects, there exist part time jobs, etc.). The participation is realized with different intensity, where usually some deal of the player's activities (time, energy, money, creativity) is located in the one-player "coalition" of his privacy. The deterministic TU-game model recollected in Section 2 ignores this multilaterality of interests and in this sense it is not realistic.

The first attempts to modify the concept of coalition and to include certain vagueness of the structure of cooperation by means of the fuzzy set theory was done in seventieths (see, e.g., [1, 2]), and it continues (cf., [3] or [14, 11, 17]). The fuzzified model is based on the definition of fuzzy coalition as a fuzzy subset of the set $I$. It means that $I$ is the universe in the sense of Section 3, and each fuzzy coalition $K$ is characterized by its membership function $\tau_{K}: I \rightarrow[0,1]$. For the simplicity of the following text, we denote the traditional crisp coalitions by $\bar{K}, \bar{L}, \ldots$, and their membership functions $\tau_{\bar{K}}, \tau_{\bar{L}}, \ldots$, where for each player $i \in I, \tau_{\bar{K}}(i)=1$ iff $i \in \bar{K}$, and $\tau_{\bar{K}}(i)=0$ iff $i \notin \bar{K}$. Due to the notations introduced in Sections 1 and 3 we denote by $\mathcal{P}(I)$ and $\mathcal{F}(I)$ the sets of all crisp and fuzzy coalitions, respectively.

4.1.1. Systems of fuzzy coalitions. The main attention of the existing literature, starting by [1] and [2] and continuously developing (via, e.g., [3] and other works) is focused on the specificity of the concept of core and its modification in the "environment" of fuzzy cooperation. Less attention is drawn to such concepts like convexity or superadditivity (e.g., the sense of disjointness) for vague coalitions each of which regards, in some degree, all or almost all players. Certain, very elementary, attempt to deal with this topic is shown in [14, 11] and [17].

The fundamental analysis of the concept of core in the TU-games with fuzzy coalitions was presented in [1], already.

First, it is necessary to introduce an extension of the characteristic function $v$ known in the deterministic TU-games for the games with fuzzy coalitions. It means that for every fuzzy coalition $K \in \mathcal{F}(I)$, there exists a real number $v(K) \in R$ representing the expected total pay-off of $K$. Let us recall that the value $v(K)$ is crisp. If $\tau_{K}(i)=0$ for all $i \in I$ then we suppose that $v(K)=0$.

If $\tau_{K}=\left(\tau_{K}(i)\right)_{i \in I}$ is the membership function of $K$ then we may write also $v\left(\tau_{K}\right)$ instead of $v(K)$. Let us suppose that for any real $\lambda>0, v\left(\lambda \cdot \tau_{K}\right)=\lambda v\left(\tau_{K}\right)$ (note that $\lambda \cdot \tau_{K}$ in the usual sense of linear algebra need not be a membership function; being treated as a general property of positive homogeneity of real functions over $R^{n}$, the above condition is correct). Then we may extend the function $v$ on the system of all non-negative vectors $\tau=\left(\tau_{i}\right)_{i \in I}$ by setting

$$
v(\tau)=\left[\sum_{i \in I} \tau_{i}\right] \cdot v\left(\frac{\tau}{\sum_{i \in I} \tau_{i}}\right), \quad \text { where } \tau \in R_{+}^{n} .
$$


The general formula can be used for the extension of $v$ on the class of all fuzzy coalitions. Having introduced the notation, we may say that a real-valued vector $c=\left(c_{1}, \ldots, c_{n}\right) \in$ $R^{n}$ is blocked by a coalition $K$ with $\tau_{K}$ if

$$
\sum_{i \in I} \tau_{K}(i) c_{i}<v\left(\tau_{K}\right)
$$

Following [1], we may define the core of such fuzzy TU-game as the set of all vectors $c=\left(c_{i}\right)_{i \in I}$ fulfilling

$$
\begin{aligned}
& \sum_{i \in I} c_{i}=v(I), \\
& \text { for all } K \in \mathcal{F}(I), \quad \sum_{i \in I} \tau_{K}(i) c_{i} \geq v(K) .
\end{aligned}
$$

It is possible to prove the following fact (see [1], Proposition 2.1)

OBSERVATION 1. If the function $v$ is concave then the core is convex, compact and nonempty.

The present model of fuzzy cooperation is relatively well treated in the literature, and it has its priority in the context of the TU-games with vague coalitions. Nevertheless, there are several aspects which deserve more attention. They are mostly connected with the attempts to join the traditional interpretation of some concepts of the TU-game theory with their fuzzified counterparts.

First of all, the concept of the fuzzy coalition as a fuzzy subset of $I$ rather wipes off the fact that a player cooperates with some partners (more or less intensively) and does not with others. That each coalition represents a common field of interests and that even joint participation of two players in more than one coalition means that they have more than one (maybe even very different) common interests. The above conception of fuzzy coalition means that in principle, each player is member of any coalition (sometimes, with vanishing degree of membership).

Further, the fuzzy coalitions demand a specific approach to the concept of their disjointness, whenever we attempt to extend the notion of superadditivity on the TU-games with fuzzy coalitions. Paper [11] offers a brief discussion of this problem.

The last aspect which would not be omitted when the games with fuzzy coalitions are considered, is the natural relation between using deterministic and fuzzy set theoretical methods in the processing of (crisp!) pay-off of (fuzzy!) coalitions. The traditional deterministic model is based, naturally, on the additive operations with pay-offs. On the other hand, processing of fuzzy sets is usually based on the monotonicity of the relevant set functions. The finding of adequate combination of both approaches, however it is briefly mentioned in [11], remains an open field of study. Some attempt in this respect was done in [17].

In the following subsection we briefly recall a method which can be used for modelling the structure of cooperative relations in the case of fuzzy coalitions. Nevertheless, it can serve as a starting point of further considerations even regarding the disjointness of fuzzy coalitions and additivity/monotonicity relations for pay-offs of fuzzy coalitions.

4.1.2. Fuzzy extensions of coalitions. The usual and quite natural interpretation of fuzziness is that it in some sense extends the crisp concepts. In the environment of the coali- 
tions forming it means that fuzzy coalitions somehow enrich the structure of cooperative relations - we may expect some connection between fuzzy coalitions and their crisp counterparts. This connection would be reflected by the pay-offs, i.e., by the values $v(K)$, as well. Certain attempt to formulate and discuss these ideas was done in [14] and [11]. Let us briefly recall its main principles.

Keeping the notations used in Subsection 4.1.1, it is easy to see that for any fuzzy coalition $K \in \mathcal{F}(I)$ the vector $\tau_{K}=\left(\tau_{K}(1), \ldots, \tau_{K}(n)\right)$ is a point from an $n$-dimensional supercube with vertices $\tau_{\bar{K}}=\left(\bar{\tau}_{\bar{K}}(1), \ldots, \bar{\tau}_{\bar{K}}(n)\right)$ representing the crisp coalitions $\bar{K} \in$ $\mathcal{P}(I)$. Let us note that $\tau_{\bar{K}}(i) \in\{0,1\}$ for any $\bar{K} \in \mathcal{P}(I)$ and $i \in I$. Then it is very easy to verify (see $[14,11])$ the following

Observation 2. If $K \in \mathcal{F}(I)$ is a fuzzy coalition and $\tau_{K}$ its membership function then there exist crisp coalitions $\bar{K}_{1}, \bar{K}_{2}, \ldots, \bar{K}_{m} \in \mathcal{P}(I)$ and real numbers $b_{1}, b_{2}, \ldots, b_{m} \in[0,1]$ such that $b_{1}+b_{2}+\cdots+b_{m}=1$ and for all $i \in I$

$$
\tau_{K}(i)=b_{1} \tau_{\bar{K}_{1}}(i)+b_{1} \tau_{\bar{K}_{1}}(i)+b_{2} \tau_{\bar{K}_{2}}(i)+\cdots+b_{m} \tau_{\bar{K}_{m}}(i)
$$

It is also easy to show that there are several sets of crisp coalitions and real coefficients representing the same fuzzy coalition $K$ by means of convex combination (1).

Keeping the notation of Observation 2 we say that $\bar{K}_{1}, \ldots, \bar{K}_{m}, b_{1}, \ldots, b_{m}$ is a crisp representation of fuzzy coalition $K$.

This relation between crisp and fuzzy coalitions offers a possibility to formulate a rule for the pay-offs $v(K)$ in some degree covering our expectation that fuzzy coalitions extend their crisp patterns. Namely, if $K$ with $\tau_{K}$ is a fuzzy coalition and $\bar{K}_{1}, \ldots, \bar{K}_{m}$ with $\tau_{\bar{K}_{1}}, \ldots, \tau_{\bar{K}_{m}}$ and real coefficients $b_{1}, \ldots, b_{m}$ is one of its crisp representations then we denote

$$
u\left(\bar{K}_{1}, \ldots, \bar{K}_{m}, b_{1}, \ldots, b_{m}\right)=\sum_{i=1}^{m} b_{i} v\left(\bar{K}_{i}\right),
$$

and we may demand for $v(K)$ to be equal to maximum of $u\left(\bar{K}_{1}, \ldots, \bar{K}_{m}, b_{1}, \ldots, b_{m}\right)$ over all crisp representations of $K$.

OBSERVATION 3. It can be shown that there exists only one crisp representation of any crisp coalition $\bar{K}$, and it is $\bar{K}$ itself. Consequently, the above condition does not contradict the definition of $(I, v)$.

The remaining paragraphs of this subsection include a rather heuristic discussion of a topic which was not studied in the literature, except [17], and which represents an open field for further investigation (even if some marginal steps were mentioned in [11], already).

If $I=\{1, \ldots, n\}$ then we denote by $\bar{L}_{0}, \bar{L}_{1}, \ldots, \bar{L}_{N}$, where $N=2^{n}-1$ and we put, without loss of generality, $\bar{L}_{0}=\emptyset, \bar{L}_{N}=I$. If $K$ is a fuzzy coalition and $\left(\bar{K}_{1}, \ldots, \bar{K}_{m}, b_{1}\right.$, $\left.\ldots, b_{m}\right)$ its crisp representation then it is possible to define a real-valued vector

$$
\boldsymbol{\beta}_{k}=\left(\beta_{0}, \beta_{1}, \ldots, \beta_{N}\right)
$$

such that $\beta_{j} \in[0,1]$ for $j=0,1, \ldots, N, \beta_{j}=b_{k}$ if $\bar{L}_{j}=\bar{K}_{k}$, and $\beta_{j}=0$ if $\bar{L}_{j} \neq \bar{K}_{k}$ for all crisp coalitions $\bar{K}_{k}$ from the considered crisp representation of $K$. The numbers 
$\beta_{0}, \ldots, \beta_{N}$ fulfil all formal properties allowing to consider the vector $\beta_{K}$ for a membership function over the set of crisp coalitions $\left\{\bar{L}_{0}, \bar{L}_{1}, \ldots, \bar{L}_{m}\right\}$. Consequently, each fuzzy coalition may be characterized by a fuzzy subset of the class of all crisp coalitions. Each such characterization describes not only which players do cooperate but also what is the structure of such cooperation (and of the interests reflected by the vague cooperation). In this sense, the membership function $\boldsymbol{\beta}_{K}$ offers better structured and more illustrative information about the interests of the players.

Moreover, some fuzzy coalitions may have several crisp representations, each of which is characterized by its own fuzzy subset $\boldsymbol{\beta}_{K}$ of the set of all crisp coalitions. They do not differ in the degree of participation of each individual player $i \in I$ in the coalition $K$-it is always described by the value $\tau_{K}(i)$. They differ in the values $\beta_{\bar{L}_{j}}$ for those crisp coalitions $\bar{L}_{j}$ for which $i \in \bar{L}_{j}$. The choice of the vector $\boldsymbol{\beta}_{K}$ from those ones corresponding with $K$ includes more information about the character of the considered relations between the vaguely cooperating players in the fuzzy coalition $K$.

Perhaps the most attractive feature of the suggested model is the fact that the crisp coalitions are a well handled concept and the processing of their (even if fuzzy) classes can effectively use our knowledge of their properties. For example, the superadditivity of fuzzy coalitions can cause some embarrassment regarding the demand of disjointness of (fuzzy) coalitions. Intuitively, we may expect that the disjointness of vague, i.e., fuzzy, objects is to be a fuzzy property but the problem is, how to define its fuzziness. It is possible to suggest several, more or less natural, approaches to this challenge. The following appears to be intuitively acceptable.

The fuzzy disjointness is a fuzzy relation, i.e., a fuzzy subset of the set of $\mathcal{F}(I) \times$ $\mathcal{F}(I)$ with membership function $\delta: \mathcal{F}(I) \times \mathcal{F}(I) \rightarrow[0,1]$, defined for any pair of fuzzy coalitions $K, K^{\prime}$ represented by vectors of membership values $\boldsymbol{\beta}=\left(\beta_{0}, \ldots, \beta_{N}\right), \beta^{\prime}=$ $\left(\beta_{0}^{\prime}, \ldots, \beta_{n}^{\prime}\right)$, respectively. The value $\delta\left(K, K^{\prime}\right)$ represents the degree in which two fuzzy coalitions coordinate similar groups of players.

The formal definition of the values $\delta\left(K, K^{\prime}\right)$ demands some auxiliary steps specifying the activities of particular players in the structure of formed cooperation. First of all, let us define for every player $i \in I$ and every fuzzy coalition $K$ with the vector of memberships $\boldsymbol{\beta}=\left(\beta_{0}, \ldots, \beta_{N}\right)$ the concept of distribution of fuzzy participation of player $i$ in $K$ as a real-valued vector

$$
\lambda^{K}(i)=\left(\lambda_{0}^{K}(i), \lambda_{1}^{K}(i), \ldots, \lambda_{N}^{K}(i)\right) \in[0,1]^{N+1},
$$

where for any $j=0,1, \ldots, N$

$$
\lambda_{j}^{K}(i)= \begin{cases}\beta_{j} & \text { if } i \in \bar{L}_{j}, \\ 0 & \text { if } i \notin \bar{L}_{j} .\end{cases}
$$

ObSERVATion 4 . For any $i \in I$ and $K \in \mathcal{F}(I)$,

$$
\sum_{j=1}^{N} \lambda_{j}^{K}(i)=\tau_{K}(i)
$$

OBSERVATION 5. Obviously, for empty crisp coalition $\bar{L}_{0}=\emptyset$ and any $i \in I, \lambda_{j}^{\emptyset}(i)=0$ for all $j=0,1, \ldots, N$. 
Observation 6. If $\bar{L}_{j} \in \mathcal{P}(I)$ is crisp then

$$
\lambda_{j}^{\bar{L}_{j}}(i)= \begin{cases}1 & \text { if } i \in \bar{L}_{j}, \\ 0 & \text { if } i \notin \bar{L}_{j} .\end{cases}
$$

Having introduced the above auxiliary concept of the distribution of fuzzy participation of players in (fuzzy) coalitions, we can formulate the fuzzified concept of disjointness with membership function $\delta\left(K, K^{\prime}\right)$ for $K, K^{\prime} \in \mathcal{F}(I)$, and to put

$$
\delta\left(K, K^{\prime}\right)=1-\max \left[\min \left(\lambda_{j}^{K}(i), \lambda_{k}^{K^{\prime}}(i)\right): i \in I, j=0,1, \ldots, N\right] .
$$

The interpretation of this formula based on the classical fuzzy set theoretical concepts is easy. The value $\delta\left(K, K^{\prime}\right)$ represents the fuzzy logical negation of the possibility that some group of players (crisp coalition) is active in both fuzzy coalitions $K$ and $K^{\prime}$.

OBSERVATION 7 . If $\bar{K}, \bar{L}$ are crisp coalitions then

$$
\delta(\bar{K}, \bar{L})= \begin{cases}1 & \text { if } \bar{K} \cap \bar{L}=\emptyset, \\ 0 & \text { if } \bar{K} \cap \bar{L} \neq \emptyset .\end{cases}
$$

Even the superadditivity may be defined as fuzzy property represented by a fuzzy subset of the class of all TU-games with fuzzy coalitions and with the set of players $I$. Its membership function will be denoted by $\sigma$ and for a game $(I, v)$ the value $\sigma(v)$ denoting the possibility that $(I, v)$ is superadditive is defined as a possibility that each pair of fuzzy coalitions $K, K^{\prime}$, such that

$$
v\left(K \cup K^{\prime}\right)<v(K)+v\left(K^{\prime}\right)
$$

is not disjoint. In symbols, it is given by formula

$$
\begin{aligned}
\sigma(v) & =\min \left[1-\delta\left(K, K^{\prime}\right): K, K^{\prime} \text { such that } v\left(K \cup K^{\prime}\right)<v(K)+v\left(K^{\prime}\right)\right]= \\
& =1-\max \left[\delta\left(K, K^{\prime}\right): K, K^{\prime}, v\left(K \cup K^{\prime}\right)<v(K)+v\left(K^{\prime}\right)\right] .
\end{aligned}
$$

Here, $K \cup K^{\prime}$ means the usual fuzzy-set theoretical union of fuzzy subsets of $I$.

The described model of TU-game with fuzzy coalitions is open but it appears promising. It can be useful to test its potential possibilities, e.g., regarding the concepts of core, convexity, value and some others. The recent or almost recent results in this field are summarized in [17].

4.2. Fuzzy expectations of profit. In the previous subsections, we have dealt with fuzzification of the first one of the concepts forming the game. The quite evident possibility to fuzzify even the pay-offs $v(\bar{K})$ for (crisp) coalitions was ignored for a relatively long time. The first attempts to fill this gap were done in the late nineties and the basic results are summarized in [10]. They were connected with the increasing interest in fuzzy data processing and its applications (see, e.g., [8, 9, 4, 6] and many others). This basic model offers both, effectively applicable results as well as urgent topics for deeper discussion. In the next two subsections, we briefly recollect the model and discuss its possible modification avoiding some of its discrepancies.

4.2.1. Pay-off as fuzzy quantities. The approach presented in [10] and developed in some other publications is based on the principle that it is not realistic to assume that the players exactly know the expected pay-offs already in the period of negotiation, it means 
before the realization of the game. In the real cooperative situations the negotiating partners only subjectively estimate their perspectives, and the expectations of the profit are more or less vague. This vagueness can be modelled by the tools of fuzzy quantities theory.

In the model analyzed in [10] only the deterministic coalitions are considered. But their pay-offs are not characterized by crisp real numbers $v(\bar{K})$ but by fuzzy quantities $w(\bar{K}) \in \mathcal{F}(R)$ whose membership functions are denoted by $\mu_{\bar{K}}: R \rightarrow[0,1]$. The pair $(I, w)$, where $w$ is a mapping of $\mathcal{P}(I)$ in $\mathcal{F}(R)$, is called a fuzzy TU-game.

As shown in Section 3, the algebraical operations and ordering relations with crisp real numbers which are used in the classical TU-games have their analogies in the environment of fuzzy quantities. In principle, it is rational to accept a paradigm that the result of any computation with fuzzy quantities is fuzzy, and that each property derived from fuzzy values is to be vague (i.e., fuzzy), too.

In the model of TU-game with fuzzy pay-offs, the previous principles mean that, e.g., the superadditivity will be a vague property. More formally, the fuzzy superadditivity is characterized by a fuzzy subset of the class of all fuzzy TU-games. The membership of this fuzzy set will be denoted by $\sigma$ and for any fuzzy game $(I, w)$ its value is defined by

$$
\sigma(w)=\max \left[\nu_{\succeq}(w(w(\bar{K} \cup \bar{L}), w(\bar{K} \oplus w(\bar{L}))): \bar{K}, \bar{L} \in \mathcal{P}(I), \bar{K} \cap \bar{L}=\emptyset)\right] .
$$

The definition of other similar properties like fuzzy convexity is quite analogous (cf. [10]). Even the core of fuzzy TU-games becomes a fuzzy concept. Namely, it is a fuzzy subset of $R^{n}$ with membership function $\gamma_{C}: R^{n} \rightarrow[0,1]$. As explained in [10], for any vector $\boldsymbol{x} \in R^{n}$ the value $\gamma_{C}(\boldsymbol{x})$ is defined by

$$
\gamma(\boldsymbol{x})=\min \left[\nu_{\succeq}\left(w(I), \sum_{i=1}^{n} x_{i}\right), \eta(\boldsymbol{x})\right]
$$

where

$$
\eta(\boldsymbol{x})=\min \left[\nu_{\succeq}\left(\sum_{i \in \bar{K}} x_{i}, w(\bar{K})\right): \bar{K} \in \mathcal{P}(I)\right]
$$

(note that any crisp real number $r \in R$ can be treated as a special fuzzy quantity with membership function condensed into a single value; in formulas, $\mu_{r}(r)=1, \mu_{r}(x)=0$ for $x \neq r)$.

The properties of the above (and some other) concepts are analyzed in [10]. The most significant for this brief presentation can be summarized in

OBSERVATION 8. If the fuzzy pay-offs $w(K)$ are substituted by crisp values $v(K)$ then the properties of the fuzzy TU-game $(\mathrm{I}, \mathrm{w})$ turn into the usual properties of deterministic TU-game $(I, v)$; i.e., fuzzy games really represent fuzzy extensions of the deterministic model.

Even if the similarity of the classical results with the ones involving fuzziness reduced to their crisp "skeleton" is impressive, the general, really fuzzy, TU-games do not preserve some properties of the crisp games. It regards, e.g., the relation between (fuzzy) convexity and (fuzzy) core, and also some other specific problems regarding fuzzy convexity. Almost all those problems follow from the algebraical properties of fuzzy quantities. They are not 
identical with the well-known group properties of crisp real numbers. Namely, the existence of the opposite element and the distributivity are not guaranteed which discrepancy can be weakened (but not avoided) by the proper specification of the concept of fuzzy zero (or fuzzy unit) (see $[8,9]$ ). Nevertheless, their consequences for the model of fuzzy TU-games keep rather destructive, as shown by several examples in [10]. It means that the model described in this subsection is adequate to some features of TU-games with fuzzy pay-offs but it is desirable to find its modification which would be more universal. We mention it in the next subsection.

4.2.2. Fuzzy classes of crisp games. The approach described here was delt, e.g., in [16] and its modification for non-transferable utility was suggested in [15].

Let us denote by $\mathcal{V}$ the set of all crisp coalitional TU-games over the set of players $I$, and let us consider a fuzzy TU-game $(I, w)$. Then it is easy to define a fuzzy subset $\mathcal{V}_{w}$ of $\mathcal{V}$ with membership function $\pi_{w}: \mathcal{V} \rightarrow[0,1]$ such that for any game $(I, v)$ the value $\pi_{w}(v)$ is defined by

$$
\pi_{w}(v)=\min \left(\mu_{K}(v(K)): K \in \mathcal{P}(I)\right) .
$$

It represents the possibility that the pay-offs in all coalitions of $(I, w)$ will be equal to those of the crisp game $(I, v)$. In this way, the fuzzy TU-game is transformed into a fuzzy class of deterministic games $\mathcal{V}_{w}$.

ObSERVATION 9. Every fuzzy TU-game $(I, w)$ can be transformed into exactly one fuzzy class of deterministic TU-games and vice-versa.

The above transformation allows to formulate the classical game-theoretical concepts for the fuzzy games by means of analogical concepts of deterministic game model without loss of the natural vagueness.

Let us start with the superadditivity. It is still a fuzzy property represented by a fuzzy subset of the class of all fuzzy TU-games. If we denote its membership function by $\bar{\sigma}$, where for any fuzzy game $(I, w)$ the possibility that it is fuzzy superadditive is

$$
\bar{\sigma}(w)=\max \left[\pi_{w}(v):(I, v) \in \mathcal{V} \text { is superadditive }\right] .
$$

The fuzzy convexity and several other concepts can be defined in a very analogous way. Namely, it is a fuzzy property presented by fuzzy subset of the class of all fuzzy TU-games. If we denote its membership function by $\chi$ then for any $(I, w)$

$$
\chi(w)=\sup \left[\pi_{w}(v):(I, v) \in \mathcal{V} \text { is convex }\right] .
$$

The general principle of the above method is applicable for a wide scale of situations. For example, even the fuzzy core of $(I, w)$ can be defined by means of the fuzzy class $\mathcal{V}_{w}$. It is a fuzzy subset of $R^{n}$ with membership function $\bar{\gamma}_{C}$ defined for any $\boldsymbol{x} \in R^{n}$ by $\bar{\gamma}(\boldsymbol{x})=\max \left[\pi_{w}(v):(I, v) \in \mathcal{V}, \boldsymbol{x} \in C_{v}\right]$, where $C_{v}$ is the core of the game $(I, v)$.

Observation 10. If the fuzzy game $(I, w)$ is a fuzzy extension of a crisp game $(I, v)$ in the sense that for all $K \subset I, \mu_{K}(v(K))=1$ then the fuzzy superadditivity and fuzzy core of $(I, w)$ are fuzzy extensions of their crisp counterparts in $(I, v)$, i.e., if $(I, v)$ is superadditive then $\bar{\sigma}(w)=1$ and if $\boldsymbol{x} \in C_{v}$ then $\bar{\gamma}_{C}(\boldsymbol{x})=1$. It is true also for other concepts. 
OBSERVATION 11. The relation between fuzzy convexity and fuzzy core of the above type exists and it is expressed in the form of inequality

$$
\chi(w) \leq \sup \left[\bar{\gamma}_{C}(\boldsymbol{x}): \boldsymbol{x} \in R^{n}\right] .
$$

The method of the transformation of fuzzy TU-games into fuzzy classes of deterministic games presented in this subsection deserves attention. It offers a possibility to exploit the well-known and deeply elaborated results on deterministic games for effective processing of uncertainty about the expected pay-offs. It can be verified that this alternative model preserves the useful properties of deterministic games like the relation between convexity and non-emptiness of core.

5. Universal vagueness: why and how? The aim of this contribution was to summarize, even if very briefly, the main methodological approaches to the existence of uncertainty, imprecision and vagueness in real cooperative situations modelled by the theory of coalitional games with transferable utility.

We can see that there exist two separated approaches to two sources of fuzzinessin the coalition structure and in the profit expectations. These two approaches are not immediately compatible even if it can be desirable to construct only one unitary model. If we accept the paradigm that the uncertainty is a universal attribute of human activities, no matter if it follows from subjectivity of human reasoning, imprecision of our knowledge, vagueness of natural languages or from other resources. Moreover, one general model can simplify the complex processing and solving various real situations. The endeavour to construct such model has its sense.

Another problem is, how to proceed in its construction. Of course, there is a possibility mechanically connect both previous classical models. For example, to accept the model of fuzzy coalitions shown in Subsection 4.1.1 and to input the fuzzy values of the coalitional pay-offs due to Subsection 4.2.1. This procedure is formally correct but it has also several evident discrepancies. As we have seen in 4.1.1 and 4.2.1, each of both "input" methods is connected with some problems regarding, e.g., the disjointness of fuzzy coalitions or the algebraical properties of fuzzy quantities. These problems do not vanish after the combination of both approaches into a united model. In fact, they are combined in accordance with the combination of models and their effect rather increases.

Moreover, the application of the fuzzy set theoretical concepts in any originally deterministic model significantly increases its complexity and, consequently, decreases its flexibility and lucidity. Relatively simple deterministic concepts are substituted by their fuzzy counterparts each of which is accompanied by membership function and more or less complicated rules for its processing. The parallel fuzzification of both components of the TU-game model, each of them with its specific formalism, can (and probably will) result into a model, the complexity of which seriously limits its handling and the verification of its advanced properties except some very elementary ones. These arguments inspire the attempts to choose another strategy for the construction of the desired united model of uncertainty in coalitional games.

One of potentially possible but not yet tested strategies can be based on the substitution of particular fuzzy elements of the game (coalitions and pay-offs) by fuzzy classes 
of their deterministic patterns (in the ideal case by fuzzy classes of deterministic games). Partial first attempts to do so were briefly shown in Subsections 4.1.2 and 4.2.2 and also in [16]. The modification and mutual adaptation of these two particular models aiming to find a general one which can cover both of them is still a challenge for the future.

Another approach combining fuzzy pay-offs and fuzzy coalitions can be found in [17], where it naturally follows from the representation of fuzzy coalitions by fuzzy classes of crisp coalitions and from the monotonicity of their pay-offs. Results shown in [17] are recent and their research continues.

It is possible to conclude that the endeavour to fuzzify the classical model of coalitional TU-games is rational and useful for eventual applications. It also offers interesting topics for theoretical research and it can, perhaps, contribute to the understanding of bargaining and coalitions forming process.

\section{References}

[1] J. P. Aubin, Cooperative fuzzy games, Math. Oper. Res. 6 (1981), 1-13.

[2] D. Butnariu, Fuzzy games: a description of the concept, Fuzzy Sets and Systems 1 (1978), 181-192.

[3] D. Butnariu and E. P. Klement, Triangular Norm-Based Measures and Games With Fuzzy Coalitions, Kluwer, Dordrecht, 1993.

[4] D. Dubois, E. E. Kerre, R. Mesiar and H. Prade, Fuzzy interval analysis, in: Fundamentals of Fuzzy Sets, D. Dubois, H. Prade (eds.), Handcontributions of Fuzzy Sets, Vol. I, Kluwer Acad. Pub. Dordrecht, 2000, 483-581.

[5] D. Dubois and H. Prade, Fuzzy numbers: An overview, in: Analysis of Fuzzy Information, J. Bezdek (ed.), Vol. I, CRC-Press, Boca Raton, 3-39.

[6] E. E. Kerre and Xuzhu Wand, Reasonable properties for the ordering of fuzzy quantities I, II , Fuzzy Sets and Systems 118 (2001), 375-385, 387-405.

[7] R. D. Luce and H. Raiffa, Games and Decisions, J. Wiley and Sons, London-New York, 1957.

[8] M. Mareš, Computation Over Fuzzy Quantities, CRC-Press, Boca Raton, 1994.

[9] M. Mareš, Weak arithmetics of fuzzy numbers, Fuzzy Sets and Systems 91 (1997), 2, $143-154$.

[10] M. Mareš, Fuzzy Cooperative Games. Cooperation With Vague Expectations, PhysicaVerlag, Heidelberg, 2001.

[11] M. Mareš, Vague utilities in cooperative market, in: Transactions of FSSCEF'2004, St. Petersburg, Vol. I, 143-153.

[12] M. Mareš and M. Vlach, Superadditivity in general fuzzy coalition games, Kybernetika 36 (2000), 265-277.

[13] M. Mareš and M. Vlach, Fuzzy coalitional structures, in: Transactions of the 7th CzechJapan Seminar on Data Processing Under Uncertainty. TU Ostrava 2003 (not paginated).

[14] M. Mareš and M. Vlach, Alternative model of fuzzy NTU coalitional games, Kybernetika 39 (2003), 265-274.

[15] M. Mareš and M. Vlach, Fuzzy classes of coalitional games with transferable utility, Scientiae Mathematicae Japonicae 60 (2004), 269-278. 
[16] M. Mareš and M. Vlach, Fuzzy coalitional structures (Alternatives), Mathware and Soft Computing. To appear.

[17] M. Mareš and M. Vlach, Fuzzy coalitions as fuzzy classes of crisp coalitions, in: Transactions of 7th Japan-Czech Seminar on Decision Making under Uncertainty, Awaji, Yumebutain August 30 -September 2, 2004. Osaka University, School of Grad. Eng., Osaka, 2004.

[18] I. Nishizaki and M. Sakawa, Fuzzy and Multiobjective Games for Conflict Resolution, Physica-Verlag, Heidelberg, 2001.

[19] J. Rosenmüller, The Theory of Games and Markets, North-Holland, Amsterdam, 1982.

[20] J. von Neumann and O. Morgenstern, Theory of Games and Economic Behaviour, Princeton Univ. Press, Princeton, 1953.

[21] L. A. Zadeh, Fuzzy sets, Information and Control 8 (1965), 338-353. 